\title{
CONTRIBUCIÓN AL CONOCIMIENTO DEL GÉNERO OEDOGONIUM (OEDOGONIALES, CHLOROPHYTA) EN ESPAÑA. II
}

\author{
Jaume CAMBRA
}

\begin{abstract}
RESUMEN. Se presentan los resultados de un estudio florístico sobre el género Oedogonium en España. En total se han estudiado 13 localidades en las que se han identificado 14 táxones, entre los cuales cabe destacar la presencia de Oedogonium khannae f. minus, Oedogonium lopesianum y Oedogonium pratense, que constituyen tres nuevas citas para la flora algológica española. Se describen la mayoría de los táxones, así como se realizan comentarios sobre su distribución y posición taxonómica.
\end{abstract}

Palabras clave. Oedogonium, taxonomía, perifiton, fitobentos dulceacuícola.

ABSTRACT. The results of a floristic survey on the genus Oedogonium in Spain are presented. A total of 13 different sites were studied, in which 14 Oedogonium taxa were identifyed, among them Oedogonium khannae f. minus, Oedogonium lopesianum and Oedogonium pratense are new records for the spanish phycological flora. Data on the morphology of the presented taxa and comments about their distribution and taxonomy are given.

Key words. Oedogonium, taxonomy, periphyton, freshwater phytobenthos.

\section{INTRODUCCIÓN}

Como continuación a los estudios emprendidos sobre el género Oedogonium Link (Oedogoniales, Chlorophyta) en sistemas acuáticos españoles (Cambra, 1989b), en esta comunicación se presentan nuevos datos sobre la distribución e interés florístico de este género.

Los sistemas muestreados corresponden a pequeñas masas de agua estancada o al litoral de lagos y lagunas. Su distribución geográfica, un tanto azarosa, se debe a que se ha estudiado material recolectado por Montserrat Comelles durante los años 1979, 1980 y 1982, así como, material procedente de campañas de recolección propias realizadas el año 1988. Las zonas investigadas comprenden 13 localidades, situadas en el nordeste y sur-sudeste de España. A continuación se detalla para cada localidad su situación geográfica, con indicación de sus coordenadas U.T.M., algunas características ecológicas generales y su flora macrofítica y helofítica más relevante. 


\section{LOCALIDADES ESTUDIADAS}

\section{1.- LAGUNA AMARGA (Córdoba)}

Laguna esteparia situada cerca de la población de Jauja (U.T.M. 30SUG52), a $400 \mathrm{~m}$ de altitud, de forma circular y $100 \mathrm{~m}$ de diámetro, aproximadamente. La vegetación litoral está dominada por Phragmites australis, Typha sp., Juncus sp. y Tamarix africana. En el fondo de laguna destacan Chara aspera, Tolypella hispanica y Potamogeton pectinatus. Las aguas de esta laguna presentaban una conductividad de $9500 \mu \mathrm{S} . \mathrm{cm}^{-1}$, una alcalinidad de $1.0 \mathrm{meq} . \mathrm{l}^{-1}$, una concentración de cloruros de $2520 \mathrm{mg} . \mathrm{l}^{-1}$ y $3170 \mathrm{mg} . \mathrm{l}^{-1}$ de sulfatos.

\section{2.- ESTANY DE BASTURS-1 (Lleida)}

Lago cárstico situado en el término municipal de Isona (U.T.M. 31TCG36), a $570 \mathrm{~m}$ de altitud, circular, rodeado por un denso cinturón de Phragmites australis; en el agua destacan Myriophyllum spicatum, Potamogeton pectinatus, Ranunculus sp. y Utricularia vulgaris. Las aguas de este lago cárstico presentaron una oscilación térmica entre $12-24.8^{\circ} \mathrm{C}$, el pH fue bastante estable, oscilando entre 7-7.6, el oxígeno disuelto se movió entre 7.1-13.5 mg. $\mathrm{l}^{-1}$, con unas conductividades entre $391-441 \mu \mathrm{S} . \mathrm{cm}^{-1}$; estas aguas presentan una importante reserva alcalina, por lo que los valores de la alcalinidad son relativamente elevados, moviéndose entre 4.75-5.16 meq..$^{-1}$. Los cloruros oscilaron entre 9.6-30.0 mg. $\mathrm{l}^{-1}$ y los sulfatos entre 9-19 mg. $\mathrm{l}^{-1}$, lo cual indica que la sal predominante en las aguas de este lago son los bicarbonatos.

\section{3.- ESTANY DE CAPMANY II (Girona)}

Laguna freática de carácter semipermanente, situada cerca de la población de la Jonquera (U.T.M. 31TDG99), a 172 m de altitud, ligeramente contaminada, con una vegetación que corresponde al Glycerieto-Sparganion (Margalef-Mir, 1981). En esta laguna se registran conductividades del orden de $669 \mu \mathrm{S} . \mathrm{cm}^{-1}$, con unas alcalinidades situadas entre 2.87 4.7 meq..$^{-1}$, los cloruros oscilan entre $74-105 \mathrm{mg} .1^{-1}$ y los sulfatos entre $64-179 \mathrm{mg} . \mathrm{l}^{-1}$.

\section{4.- ARROYO DE LA CORCOBADA (Córdoba)}

Se trata de un charco cercano al arroyo de aguas muy puras y fondo turboso, con Fontinalis antipyretica, Hippuris vulgaris, Callitriche sp., Isoetes $\mathrm{sp} .$, Ranunculus $\mathrm{sp} .$, Juncus sp. y Glyceria fluitans.

\section{5.- LAGUNA DE GUARDAFUEGOS (Huelva)}

Laguna de aguas limpias, de carácter temporal sobre substrato arenoso, situada cerca de la población de Moguer, en el camino de Marzagón a Matillas (U.T.M. 29SPB91). La vegetación está constituída por helófitos bajos como Schoenus nigricans y Alisma ranunculoides y macrófitos como Myriophyllum alterniflorum y Ranunculus sp. Las aguas de esta laguna registran un $\mathrm{pH}$ de 6.6 , una alcalinidad de 0.53 meq. $1^{-1}$, una concentración de cloruros de 29 $\mathrm{mgl}^{-1}$ y de $14 \mathrm{mg} \cdot \mathrm{l}^{-1}$ de sulfatos.

\section{6.- LAGUNA DEL MAJÓN (Cáceres)}

Situada en el término municipal de la Malpartida de Cáceres (U.T.M. 29SQD17), de unos $300 \mathrm{~m}$ de diámetro, aproximadamente; con una vegetación dominada por helófitos bajos comoJuncus sp. y macrófitos como Ranunculus sp., Mentha sp., Callitriche sp. En esta laguna registramos un $\mathrm{pH}$ de 7 , una alcalinidad de 0.45 meq..$^{-1}$, una concentración de cloruros de 38 mg. $l^{-1}$ y de $8 \mathrm{mg} . \mathrm{l}^{-1}$ de sulfatos.

\section{7.- LAGUNA DE LA MASSONA (Girona)}

Laguna litoral cercana a la desembocadura del río Fluvià (U.T.M. 31TEG07), a nivel del mar, de silueta alargada y perpendicular al mar; rodeada por una densa vegetación de Phragmites australis. Durante el período de muestreo se registró una variación de la temperatura del agua entre $16-26.7^{\circ} \mathrm{C}$, el pH osciló entre 7.3-8.2, el oxigeno disuelto entre 5.4-10.4 mg..$^{-1}$, la conductividad entre $951-4110 \mu \mathrm{S} . \mathrm{cm}^{-1}$, la alcalinidad entre $2.08-5.46$ meq..$^{-1}$, los cloruros entre 200-1930 mg..$^{-1}$ y los sulfatos entre $48-300 \mathrm{mg} \cdot \mathrm{l}^{-1}$. 


\section{8.- LAGUNA DE LAS MESILLAS (Cáceres)}

Situada cerca de la población de Almaraz (U.T.M. 30STK70), a $380 \mathrm{~m}$ de altitud, de unos $3 \mathrm{~m}$ de diámetro y de carácter temporal. En esta laguna se registró una temperatura de $15^{\circ} \mathrm{C}$, una conductividad de $58 \mu \mathrm{S} . \mathrm{cm}^{-1}$ y una alcalinidad de 0.17 meq. $1^{-1}$.

9.- RÍO NOGUERA PALLARESA (Lleida)

Charco cercano al río, en el término municipal de Tremp (U.T.M. 31TCG27).

\section{0.- LAGUNA DEL REMOLAR (Barcelona)}

Laguna litoral, situada cerca de la desembocadura del río Llobregat (U.T.M.31TDF27), alterada por la actividad antrópica.

\section{1.- LAGUNA DE ST. MIQUEL (Lleida)}

Laguna temporal cercana al Puig de Sant Miquel (31TCG16), a $1060 \mathrm{~m}$ de altitud. La laguna ocupa una depresión sobre un substrato rocoso de tipo calcáreo; se alimenta exclusivamente del agua de las precipitaciones. Todo el fondo de la cubeta está ocupado por Chara hispida y en el litoral predomina Typha sp. En esta laguna la temperatura del agua osciló entre 5.7-28.7 ${ }^{\circ} \mathrm{C}$, el pH entre 7.6-8.4, el oxígeno disuelto entre $6.9-14 \mathrm{mg} . \mathrm{l}^{-1}$, la conductividad entre 140-294 $\mu \mathrm{S} . \mathrm{cm}^{-1}$, la alcalinidad entre 2.32-3.07 meq. $\mathrm{l}^{-1}$, los cloruros entre $1-7 \mathrm{mg} . \mathrm{l}^{-1}$ y los sulfatos entre $3.2-19.8 \mathrm{mg} . \mathrm{l}^{-1}$.

\section{2.- EMBALSE DE VALL-LLOSSERA (Barcelona)}

Embalse construido en los años cuarenta, en el término municipal de Hostalets de Balenyà (U.T.M. 31DG3729), a $600 \mathrm{~m}$ de altitud, enclavado sobre un substrato calcáreo; con una vegetación macrofítica dominada por Potamogeton pusillum, Utricularia vulgaris y Chara vulgaris (Cambra y Perera, 1989).

\section{3.- LAGUNA DE VILAÜT (Girona)}

Laguna semipermanente, poco profunda, situada cerca de la población de Castelló d'Empúries (U.T.M. 31TEG0982), a unos $10 \mathrm{~m}$ de altitud, con una vegetación dominada por Iris pseudacorus, Carex sp., Eleocharis palustris, Oenanthe fistulosa, Ranunculus sp. y Callitriche sp. En esta laguna se registró una variación anual de la temperatura entre 14$22.7^{\circ} \mathrm{C}$, el pH osciló entre 7.2-7.8, el oxígeno disuelto entre $2.8-6.8 \mathrm{mg} .1^{-1}$, la conductividad entre 1130-1743 $\mu \mathrm{S} . \mathrm{cm}^{-1}$, la alcalinidad entre 6.27-8.24 meq. $\mathrm{l}^{-1}$, los cloruros entre 206-492 mg..$^{-1}$ y los sulfatos entre $129-396 \mathrm{mg} \cdot \mathrm{l}^{-1}$.

\section{METODOLOGÍA}

Las muestras se han recolectado directamente desde el litoral de los sistemas estudiados, siendo fijadas inmediatamente con formaldehído al 4\%. En el laboratorio, el material se ha estudiado bajo la lupa binocular y posteriormente al microscopio. En cada caso, solamente se han tenido en consideración aquellos filamentos que presentaban oósporas en óptimo estado de maduración. Los ejemplares se encuentran depositados en el herbario del Depto. de Biología Vegetal, Fac. Biología, Universidad de Barcelona, bajo la denominación BCC-JC. En cada campaña de recolección se han recogido algunos datos físico-químicos del agua, analizándose in situ la temperatura y el oxígeno disuelto (Oxímetro WTW), pH (PH-metro CRISON) y conductividad (Conductímetro CRISON). Así mismo, se fijaron muestras de agua con unas gotas de cloroformo con la finalidad de analizar en el laboratorio los siguientes parámetros: alcalinidad (valoración potenciométrica Autotitulador METRHOM), cloruros y sulfatos (Cromatografía iónica).

Para la identificación de los táxones recolectados se han utilizado las siguientes monografías: Lacerda (1946), Mrozinska (1985), Gonzalves (1981), Jao (1979), Gemeinhardt (1939) y Gauthier-Lièvre (1963-64). 


\section{FLORA}

En total se han registrado 14 táxones, de los cuales Oedogonium khannae f. minus, Oedogonium pratense y Oedogonium lopesianum corresponden a nuevas citas para la flora algológica española.

Oedogonium borisianum (Le Clerc) Wittrock (Lám. I; Fig. 1)

Monoico, nanándrico. Filamentos formados por células de 62-80 $\mu \mathrm{m}$ de largo por 12$13 \mu \mathrm{m}$ de ancho. Oogonios de $62-63 \mu \mathrm{m}$ de largo por $40 \mu \mathrm{m}$ de ancho, cilíndricos, con la parte apical engrosada. Oósporas de $50 \mu \mathrm{m}$ de largo por 37-40 $\mu \mathrm{m}$, más o menos esféricas, con el mesosporio liso. Androsporocistes de $10 \mu \mathrm{m}$ de largo por 7.5-8.0 $\mu \mathrm{m}$ de ancho. Nanandros pluricelulares, nanandrospermatocistes de $25 \mu \mathrm{m}$ de largo por $12.5 \mu \mathrm{m}$ de ancho.

Localidad: Laguna del Majón, 17/04/1979; Charco cercano al río Noguera Pallaresa, 29/08/1980.

Hábitat: Entre el plocon litoral.

BCC-JC: $1048,1051$.

Leg.: M. Comelles.

Observaciones: A pesar de ser una especie cosmopolita (Gemeinhardt, 1939; GauthierLièvre, 1963-64; Jao, 1979; Mrozinska, 1985) en España se ha citado en escasas ocasiones (Allorge y Allorge, 1930; Cambra, 1989a), también se ha observado en Portugal (Lacerda, 1946), Eslovenia (Lazar, 1960), Francia, Argelia, Marruecos y Túnez (Gonzalves, 1981).

Oedogonium capillare (L.) Kützing, 1843

Loc.: Laguna del Remolar, primavera/1982

Hab.: Entre el plocon litoral.

BCC-JC: 1052.

Leg.: X. Millet

Obs.: Cosmopolita (Gemeinhardt, 1939; Mrozinska, 1985; Jao, 1979). En el estado español ha sido poco citado (Cambra, 1989b; Lewin, 1888; Margalef, 1956a,b; Sabater, 1987), en cambio se ha encontrado con frecuencia en otros países de la cuenca mediterránea, como Argelia (Gauthier-Lièvre, 1963-64), Libia, Marruecos, Francia, Italia y Yugoslavia (Gonzalves, 1981). Su amplia distribución la atribuimos a la capacidad de esta especie de vivir en diversos medios acuáticos, tanto alcalinos como ligeramente salobres, comportamiento que explica su gran difusión en un río muy mineralizado como el Ter.

Oedogonium crispum (Hassall) Wittrock, 1874

(Syn.: Oedogonium hispanicum Lewin sensu Gonzalves, 1981)

Monoico, macrándrico. Células de $57-60 \mu \mathrm{m}$ de largo por $10-15 \mu \mathrm{m}$ de ancho. Oogonios de 38-40 $\mu \mathrm{m}$ de largo por $42 \mu \mathrm{m}$ de ancho, solitarios, globosos o piriformes, con una fisura superior. Oósporas de 33-34 $\mu \mathrm{m}$ de diámetro, esféricas. Episporio y mesosporio lisos. Espermatocistes de 7-10 $\mu \mathrm{m}$ de largo por 10-11 $\mu \mathrm{m}$ de ancho, en séries de 2-3 y situados a continuación de la célula epígina.

Loc.: Laguna del Majón, 17/04/1979; Estany de Basturs-1, 23/07/1988

Hab.: Entre el epifiton de Callitriche sp., Ceratophyllum demersum y Myriophyllum spicatum.

BCC-JC: 916, 917, 1048.

Leg.: M. Comelles, J. Cambra.

Obs.: Es una de las pocas especies del género Oedogonium que pueden tolerar una ligera salinidad. Cosmopolita.

Oedogonium gunnii Wittrock, 1874

Loc.: Embalse de Vall-Llossera, 12/06/1988.

Hab.: Entre el epifiton de Callitriche sp. y Utricularia vulgaris.

BCC-JC: 1020.

Obs.: Muy difundido, aunque, casi siempre se encuentran filamentos fértiles con oósporas sin madurar. Citado en América (Dillard, 1989; Pereira y Parra, 1984), China (Jao, 


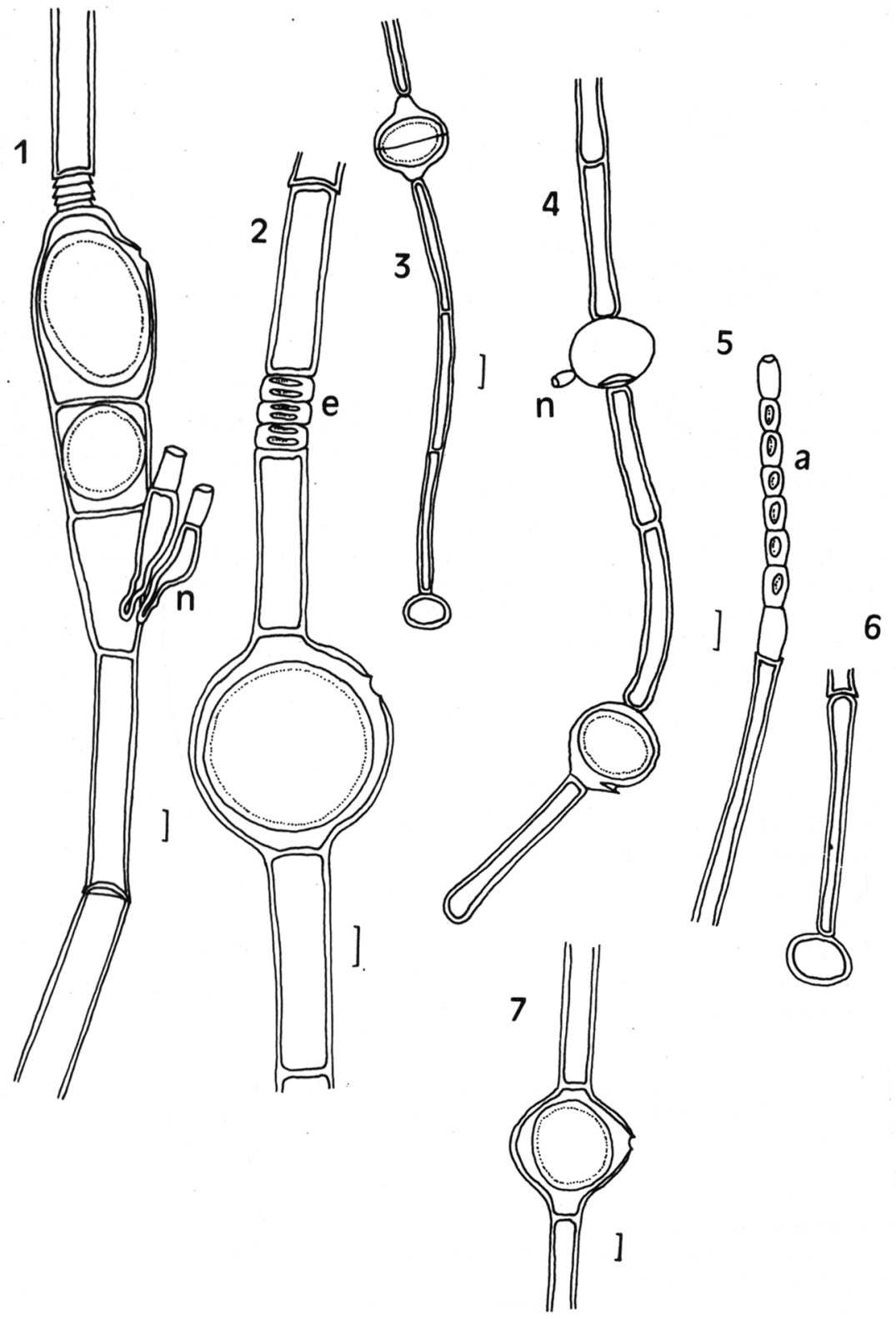

Lámina I. Fig. 1, Oedogonium borisianum (Le Clerc) Wittrock; n: Nanandrospermatocistes. Fig. 2, Oedogonium intermedium Wittrock; e: Espermatociste. Fig. 3, Oedogonium khannae Skuja f. minus Gonzalves y Jain. Figs. 4-6, Oedogonium lopesianum Lacerda.; a: Androsporocistes; n: Nanandro. Fig. 7, Oedogonium rufescens Wittrock. (Todos los segmentos equivalen a $10 \mu \mathrm{m}$ ). 
1979) y países mediterráneos como Argelia (Gauthier-Lièvre, 1963-64), Egipto (Gonzalves, 1981). En España solamente se había citado en el nor-oeste peninsular (Margalef, 1956a).

Oedogonium intermedium Wittrock in Wittrock y Nordstedt, 1886 (Lám. I; Fig. 2)

Monoico, macrándrico. Células de $50 \mu \mathrm{m}$ de largo por $12.5-15.0 \mu \mathrm{m}$ de ancho, cilíndricas. Oogonios de 40-44 $\mu \mathrm{m}$ de diámetro, esféricos o ligeramente ovoides o incluso cordiformes, con un poro superior. Oósporas de 37-38 $\mu \mathrm{m}$ de diámetro, esféricas. Episporio y mesosporio lisos. Espermatocistes de 6-7.5 $\mu \mathrm{m}$ de largo por $12-15 \mu \mathrm{m}$ de ancho, en séries de 2-3 y con dos espermatozoides dispuestos de manera horizontal.

Loc.: Laguna de la Massona, 20/07/1988; Laguna Amarga, 11/04/1982.

Hab.: Entre el epifiton de Chara aspera, P hragmites australis y Potamogeton pectinatus.

BCC-JC: $968,1041$.

Leg.: M. Comelles, J. Cambra.

Obs.: Oedogonium fragile y $O$. globosum son dos especies muy similares a $O$. intermedium, ya que presentan formas o razas intermedias mal delimitadas. Posiblemente $O$. globosum y $O$. intermedium són razas de $O$. fragile, con una morfometría ligeramente inferior. No obstante, los ejemplares estudiados presentan unas dimensiones intermedias, las cuales se podrían atribuir a cualquiera de éstas espécies.

O. intermedium es cosmopolita (Dillard, 1989; Jao, 1979; Prescott, 1951), manifiesta una cierta preferencia por las aguas salobres de carácter oligohalino (Gemeinhardt, 1939), apreciación que coincide con nuestros datos ecológicos. En la península Ibérica, solo ha sido citado en Mallorca (Margalef, 1953b) y en el sud-este peninsular (Aboal, 1988). En otros países de la cuenca mediterránea solamente se conoce de Argelia (Gauthier-Lièvre, 1963-64) y Egipto (Gonzalves, 1981).

\begin{tabular}{lccc}
\hline & 1 & 2 & 3 \\
\hline Diámetro celular & $5-10$ & $8-14$ & $10-13$ \\
Longitud celular & $50-90$ & $20-65$ & $82-110$ \\
Diámetro oogonio & $27-35$ & $28-35$ & $32-40$ \\
Longitud oogonio & $20-30$ & $23-38$ & $27-30$ \\
Diámetro oóspora & $25-33$ & $23-34$ & 33 \\
Longitud oóspora & $18-28$ & $21-30$ & 28 \\
Diámetro androsporangio & $5-8$ & $8-9$ & 10 \\
Longitud androsporangio & $12-18$ & $6-10$ & $7-12$
\end{tabular}

Tabla I. Variación morfométrica (valores extremos) de 1: Oedogonium decipiens var. dissimile y 2: O. lopesianum (Gonzalves, 1981; Mrozinska, 1985) y 3: ejemplares estudiados de $O$. lopesianum.

Oedogonium itzigsohnii De Bari, 1854

Monoico. Macrándrico. Filamentos formados por células de $10 \mu \mathrm{m}$ de diámetro por 26$30 \mu \mathrm{m}$ de largo. Oogonios solitarios, de $34 \mu \mathrm{m}$ de diámetro por $36 \mu \mathrm{m}$ de largo, elipsoidales, con ocho o nueve protuberancias gibosas situadas en la parte media del oogonio, fisura ecuatorial. Oóspora de $22 \mu \mathrm{m}$ de diámetro, con el mesosporio liso.

Loc.: Laguna de Guardafuegos, 16/05/1979.

Hab.: Epifítico de Myriophyllum alterniflorum.

BCC-JC: 1046.

Obs.: Se trata de una especie propia de hábitats turbosos (Gemeinhardt, 1939). En España solamente se había encontrado en el nor-oeste (Margalef, 1956a; Allorge y Allorge, 1930). 
Oedogonium khannae Skuja, 1949 f. minus Gonzalves y Jain, 1968 (Lám. I; Fig. 3)

Dioico, macrándrico. Células de 20-22 $\mu \mathrm{m}$ de largo por (2.5)-4 $\mu \mathrm{m}$ de ancho, cilíndricas, rectas o ligeramente curvadas, más o menos capiteladas. Célula basal de 10-12 $\mu \mathrm{m}$ de diámetro, elíptica. Oogonios de 18-19 $\mu \mathrm{m}$ de largo por 15-16 $\mu \mathrm{m}$ de ancho, elípticos, solitarios o en séries de 2-3, con una fisura ecuatorial. Oósporas de $12 \mu \mathrm{m}$ de largo por $15 \mu \mathrm{m}$ de ancho, elípticas. Episporio y mesosporio lisos.

Loc.: Laguna de las Mesillas, 03/1982; Laguna de Sant Miquel, 24/04/1988, 24/05/ $1988,29 / 06 / 1988$.

Hab.: Entre el epifiton de Chara hispida.

BCC-JC: 927, 928, 929, 1055.

Obs.: Especie raramente citada (Gonzalves y Jain, 1968).

Oedogonium lopesianum Lacerda, 1949 (Lám. I; Figs. 4, 5, 6)

Nanándrico, idioandrospórico. Células vegetativas de 82-110 $\mu \mathrm{m}$ de largo por 10-13 $\mu \mathrm{m}$ de ancho, cilíndricas o ligeramente capiteladas. Célula basal de $50 \mu \mathrm{m}$ de largo por 12.5 $\mu \mathrm{m}$ de ancho, alargada, claviforme. Oogonios de 27-30 $\mu \mathrm{m}$ de largo por 32-40 $\mu \mathrm{m}$ de ancho, elípticos. Oósporas de $28 \mu \mathrm{m}$ de largo por $33 \mu \mathrm{m}$ de ancho. Episporio y mesosporio lisos. Androsporocistes de 7.5-12.5 $\mu \mathrm{m}$ de largo por $10 \mu \mathrm{m}$ de ancho, en séries de hasta ocho, con un solo gámeta. Nanandros unicelulares, de $7.5 \mu \mathrm{m}$ de diámetro, situados en la célula epígina o sobre los oogonios.

Loc.: Laguna de Sant Miquel, 24/05/1988, 29/06/1988.

Hab.: Muy abundante entre el plocon litoral y entre el epifiton de Chara hispida.

BCC-JC: $928,929$.

Obs.: El material examinado encaja en líneas generales con $O$. lopesianum. Por los datos morfológicos de que disponemos se podría situar también en el grupo de Oedogonium decipiens, en particular afin a su var. dissimile (Hirn) Tiffany. La morfometría (Tabla I) es similar en los tres casos, la diferencia estriba en que mientras los oogonios de $O$. decipiens var. dissimile se abren mediante una fisura ecuatorial, los de $O$. lopesianum lo hacen mediante un poro alargado en situación basal, rasgo que coincide con nuestro material. Oedogonium lopesianum solamente se ha citado en Portugal (Lacerda, 1949).

Oedogonium macrandrium Wittrock, 1870 f. lundense (Wittrock) Hirn, 1906

Loc.: Estany de Capmany II, 13/05/1979.

Hab.: Entre el plocon litoral.

BCC-JC: 1049 .

Leg.: M. Comelles.

Obs.: Taxon raro, solo se conoce de Dinamarca (Gemeinhardt, 1939), Finlandia, Francia y Suecia (Gonzalves, 1981), Estados Unidos (Dillard, 1989; Prescott, 1951) y norte de Africa (Gauthier-Lièvre, 1963-64). En España solamente se había encontrado en el cap de Creus, Girona (Cambra, 1989a) y en la laguna de Vilaüt, Girona (Cambra, 1989b).

Oedogonium oblongum Wittrock, 1872

Loc.: Laguna de Guardafuegos, 16/04/1979.

Hab.: Epifítico de Myriophyllum alterniflorum.

BCC-JC: 1046.

Leg.: M. Comelles.

Obs.: En España solamente se ha citado en charcos temporales del Garraf (Cambra, 1989b). También se conoce de otros países de la cuenca mediterránea como Argelia (GauthierLièvre, 1963-64), Libia y Marruecos (Gonzalves, 1981).

Oedogonium pratense Transeau, 1914 (Lám. I; Fig. 4)

Dioico, macrándrico. Células de 51-52 $\mu \mathrm{m}$ de largo por 9-10 $\mu \mathrm{m}$ de ancho. Oogonios de 25-30 $\mu \mathrm{m}$ de diámetro, elípticos, con una fisura ecuatorial, solitarios o en parejas. Oósporas de 22-23 $\mu \mathrm{m}$ de diámetro, elípticas. Mesosporio liso.

Loc.: Laguna de Vilaüt, 20/04/1988.

Hab.: Epifítico de Oenanthe fistulosa.

BCC-JC: 923.

Obs.: Especie poco citada, solamente se conoce de Asia (Gonzalves, 1981), América, Alemania, Suecia (Gemeinhardt, 1939) y Argelia (Gauthier-Lièvre, 1963-64). 
Oedogonium quintanilhae Lacerda, 1946

Monoico, nanándrico. Filamentos formados por células de $250 \mu \mathrm{m}$ de largo por $25 \mu \mathrm{m}$ de ancho. Oogonios de $75 \mu \mathrm{m}$ de largo por 57-58 $\mu \mathrm{m}$ de ancho, subesféricos, terminales o intercelulares. Oósporas de $62-63 \mu \mathrm{m}$ de largo por $50 \mu \mathrm{m}$ de ancho, oblongas. Mesosporio escrobiculado, con las fosetas dispuestas sin orden. Nanandrospermatocistes de $25 \mu \mathrm{m}$ de largo por $12-13 \mu \mathrm{m}$ de ancho.

Loc.: Laguna del Majón, 17/04/1979.

Hab.: Entre el plocon litoral, sobre Callitriche sp. y Ranunculus sp.

BCC-JC: 1048 .

Obs.: En la península Ibérica solo se conocía de Portugal (Lacerda, 1946) y del cap de Creus, Girona (Cambra, 1989a).

Oedogonium rufescens Wittrock, 1870 (Lám. I; Fig. 7)

Dioico, macrándrico. Filamentos formados por células de $42-50 \mu \mathrm{m}$ de largo por 12$13 \mu \mathrm{m}$ de ancho. Oogonios de $25-30 \mu \mathrm{m}$ de largo por $25 \mu \mathrm{m}$ de ancho, oblongos a elipsoidales, con un poro ecuatorial. Oósporas de 22-25 $\mu \mathrm{m}$ de diámetro, esféricas. Mesosporio liso.

Loc.: Laguna del Majón, 17/04/1979.

Hab.: Epifítico de Callitriche sp. y Ranunculus sp.pl.

BCC-JC: 1048

Obs.: Especie relativamente difundida (Gemeinhardt, 1939). En España ha sido citada por Margalef (1953a). También se conoce de Argelia (Gauthier-Lièvre, 1963-64), Portugal, islas Azores y Yugoslavia (Gonzalves, 1981).

Oedogonium suecicum Wittrock var. peregrinum Skuja, 1964

Dioico, macrándrico. Filamentos de 17-18 $\mu \mathrm{m}$ de diámetro. Oogonios de $45 \mu \mathrm{m}$ de largo por $42-43 \mu \mathrm{m}$ de ancho, subesféricos, con un poro supra-ecuatorial. Oósporas de $35 \mu \mathrm{m}$ de diámetro, esféricas. Mesosporio espinoso, con espinas de hasta $2.5 \mu \mathrm{m}$ de largo. 04/1979.

Loc.: Charco en el arroyo de la Corcobada, 10/04/1982; Laguna de Guardafuegos, 16/

Hab.: Epifítico de Fontinalis antipyretica y Myriophyllum alterniflorum.

BCC-JC: 1046, 1047.

Leg.: M. Comelles.

Obs.: En España solamente se había citado en el cap de Creus, Girona (Cambra, 1989a).

AGRADECIMIENTOS. Expreso mi agradecimiento a Montserrat Comelles, por la amable cesión de buena parte del material estudiado, tanto a nivel florístico como de algunos datos físico-químicos. También hago extensivo mi agradecimiento a los miembros del Servicio de Espectroscopia de la Univ. de Barcelona por la realización de los análisis de cloruros y sulfatos. Así mismo, hago constar que este trabajo se ha realizado con una beca de F.P.I. del Ministerio de Educación y Ciencia.

\section{BIBLIOGRAFIA}

ABOAL, M. -1988- Aportación al conocimiento de las algas epicontinentales del SE de España III. Clorofíceas (Chlorophyceae Wille in Warming, 1884). Candollea, 43: 521 548.

ALLORGE, P. Y V. ALLORGE -1930- Hétérocontes, Euchlorophycées et Conjuguées de Galice. Rev. Algol., 5: 327-382.

CAMBRA, J. -1989a- Freshwater algae from the cape of Creus peninsula, Catalonia (N.E. Spain). Nova Hedwigia, 49(3-4): 281-321.

CAMBRA, J. -1989b- Contribución al conocimiento del género Oedogonium en España I. Acta Bot. Malacitana, 14: 201-206.

CAMBRA, J. y M. PERERA -1989- Nota sobre la flora acuática de los embalses de Vall- 
Llossera (Barcelona, España). An. Biol., 15(4): 131-134.

DILLARD, G.E. -1989- Freshwater algae of the Southeastern United States. Bibl. Phycol., 83: $1-163$

GAUTHIER-LIÈVRE, L. -1963-64- Oedogoniacées Africaines. Nova Hedwigia, 6-7: 151$481 ; 545-558$.

GEMEINHARDT, K. -1939- Oedogoniales. In: Rabenhorst's Kryptogamenflora von Deutschland, Österreich und Schweitz. Leipzig.

GONZALVES, E.A. -1981- Oedogoniales. I.C.A.R. Ed. New Delhi.

GONZALVES, E.A. \& S.C. JAIN -1968- Some Oedogoniaceae from Tungar Hill and surrounding areas. Phycos, 7: 9-22.

JAO, C.C. -1979- Monographia Oedogoniales Sinicae. Sc. Press. Ed. Beijing.

LACERDA, F.S. -1946- Oedogoniaceae de Portugal. Portug. Acta Biol., 2: 1-159.

LACERDA, F.S. -1949- Oedogoniaceae de Portugal II. Portug. Acta Biol.: 36-54.

LAZAR, J. -1960- Alge Slovenije. Acad. Sci. Art. Slovenica, 10.

LEWIN, M. -1888- Über spanische Süsswasseralgen. Bih. Svensk. Vetensk. Akad. Handl., 14: $1-24$.

MARGALEF, R. -1956a- Algas de agua dulce del noroeste de España. Publ. Inst. Biol. Apl., 22: 43-152.

MARGALEF, R. -1956b- Materiales para una flora de las algas del NE de España VII. Suplemento. Coll. Bot., 5(1): 87-107.

MARGALEF-MIR, R. -1981- La vegetación vascular de las aguas dulces y salobres del N.E. y litoral mediterráneo de España: Distribución y dependencia de la composición química del medio. Memoria Fundación J. March. Barcelona.

MROZINSKA, T. -1985-Oedogoniales. In: ETTL, H. et al. (Eds.) Süsswasserflora von Mitteleuropa. Stuttgart.

PEREIRA, I.A. y O.O. PARRA -1984- Algas filamentosas dulceacuícolas de Chile I. Algas bentónicas de Concepción. Gayana Bot., 41: 141-200.

PRESCOTT, G.W. -1951-Algae of the western great lakes area. O. Koeltz Sc. Ed. Koenigstein. SABATER, S. -1987- Estudi de les poblacions d'algues del riu Ter. Tesis Doctoral. Univ. Barcelona. 